\title{
Six views of the Universe
}

\section{Harry Shipman and Sharon Juska}

Creation: The Story of the Origin and Evolution of the Universe. By Barry Parker. Plenum:1988. Pp.297. \$22.95.

The Dark Side of the Universe. By James Trefil. Scribners:1988. Pp.197. \$17.95.

The Big Bang, revised and updated edition. By Joseph Silk. W. H. Freeman:1988. Pp.485. Hbk £19.95, \$24.95; pbk £9.95, $\$ 13.95$.

Gravity's Lens: Views of the New Cosmology. By Nathan Cohen. Wiley:1988. Pp.237. \$19.95, £12.95.

The Supernova Story. By Laurence A Marschall. Plenum:1988. Pp.296. \$22.95. Relatively Speaking: Relativity, Black Holes, and the Fate of the Universe. By Eric Chaisson. W. W. Norton:1988. Pp. 254. \$18.95.

FOR astronomers, the past few years have been an exciting time. What seemed to be a virtually complete picture of the evolution of the Universe in the late 1970s has been transformed by the connection between particle physics and cosmology. This 'inner space/outer space' connection has spawned many exciting ideas, such as the hypothetical 'dark matter' and the origin of large-scale structure in the Universe. When an obscure blue star called Sanduleak-69202 exploded in the Large Magellanic cloud, its violent death throes sent shock waves through its immediate vicinity, lighting up neighbouring gas clouds. Some 160,000 years later, this supernova sent shock waves through a community of astronomers living far away on Earth, dramatically confirming our ideas on how massive stars die in this most spectacular of celestial events. And then there remains a curious galaxy of astronomical beasts such as black holes, energetically interacting binary systems like SS 433 (the only star which had a finding chart shown on the Saturday Night Live television show), and gravitational lenses.

There are far more popular books on astronomy than on other scientific subjects, and still they come. The half dozen reviewed here, all addressed to the proverbial 'layperson', largely succeed in communicating astronomy's excitement both to scientists in other fields and to the public at large. All that is asked of the reader is a tolerance for astronomically large or small numbers and for a few strange uses of language. For instance, astrophysicists use the word 'inflation' to describe an abrupt increase in the size of the Universe which occurred $10^{-3.4}$ seconds after the Big Bang, not rising prices.

Four of the books deal to a greater or lesser degree with cosmology, the evolu- tion of the Universe. The emphasis on 'classical', well-established research and on contemporary work which may be more speculative varies. The most classical of the four is Barry Parker's Creation, a well-conducted tour through the standard Big Bang theory spiced with just a bit of dark matter and such. It is now quite well established that the Universe did have a hot beginning some 10 to 20 billon years ago, and it is this idea rather than contemporary embellishments that Parker primarily concentrates on.

Parker is particularly good at bringing scientists to life. His descriptions of Hubble's brief forays into boxing, Gamow's impish sense of humour and boundless energy, Mike Turner's two-

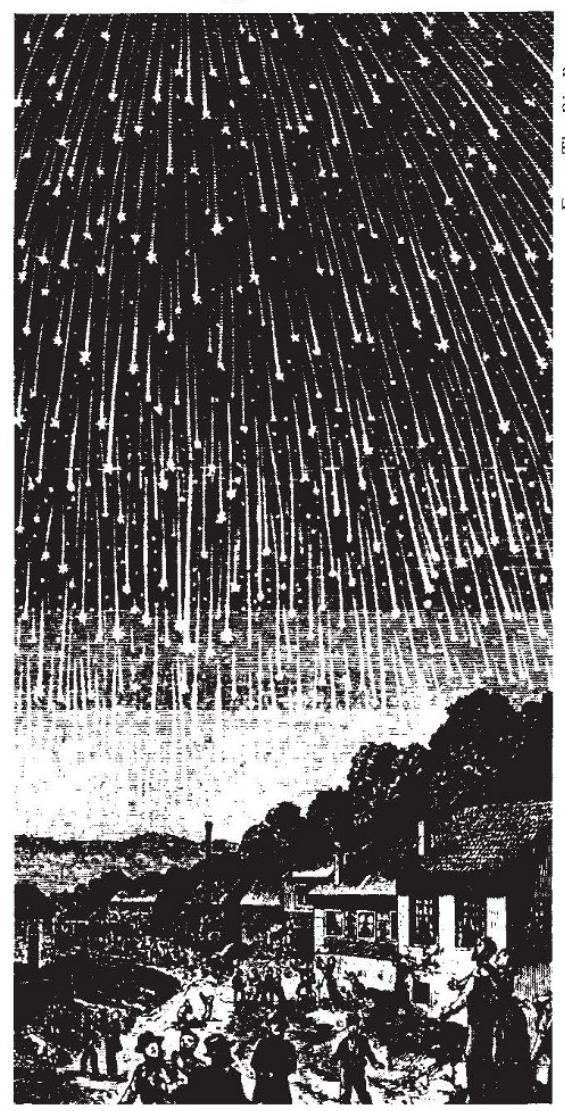

Catch a falling star - the Leonid meteor shower of November 1833, which appears every 33 years.

year career as a car mechanic and other personal vignettes are marvellous. The illustrations complement the text well. Parker includes, for instance, the splendid picture of Gamow emerging from a bottle of Cointreau labelled as "YLEM" Gamow's term for the primordial material.

Occasional organizational slips (black holes are stuffed in for no good reason) and infrequent lapses into leaden prose detract in only a minor way from what is generally an excellent treatment. Authors of books such as this always have a problem in distilling our understanding of particle physics into one chapter, but Parker meets the challenge, presenting a picture which was clear to the one of us who had never encountered particle physics before
(S.J.) and was still illuminating to the other (H.S.).

James Trefil covers much the same ground as Parker, but with greater emphasis on current controversies. Trefil's sceptical, even irreverent attitude towards currently fashionable cosmology is a magnificent antidote to the information-dump style of science textbooks and even some popular books. Trefil even includes a little table where he estimates the probability that various ideas are correct. He gives the basic Big Bang theory a 99 per cent plus chance of being right, while hypothetical yet popular superstrings are rated at 20 per cent. The main topic dealt with in the book is 'dark matter', a sea of particles of currently unknown nature which, according to current prejudices, should make up at least 90 per cent of the contents of the Universe and play a key role in the formation of galaxies. There is, too, a thought-provoking section on how understanding the evolution of the Universe does not mar our appreciation of its beauty, a defence of the scientific approach to cosmology.

Trefil's sense of humour comes through in chapter titles such as "Five Reasons Why Galaxies Can't Exist". At one point, in the middle of a difficult discussion of supersymmetry, he asks the reader directly, "are you sure you don't want to skip ahead to the summary on page 255 ?". Those who find the 10- and 26-dimensional worlds postulated by some modern particle theorists incomprehensible, incredible or both can find some comfort here.

Joseph Silk's The Big Bang, originally published in 1980, has now emerged in revised and updated form. This book, by far the longest and most intellectually challenging of the six reviewed here, is not really for the same audience as the others, even though all equations are consigned to a 40-page mathematical appendix. People with no science background can certainly get a great deal from much of the text, and can appreciate the more thorough treatment of the philosophical and mathematical foundations of the subject. But it is not always easy going, and some parts such as the three-chapter section on galaxy formation, evolution and clustering may be out of reach of strictly lay readers. This book is drier and less personal than the others; for example, the story of how Alan Guth discovered inflation, a science classic comparable to the story of Archimedes running naked through the streets, crying "Eureka", is all subsumed in the two words "brilliant insight".

But length and depth also have their virtues. For example, the history of cosmological thought is carried back as far as Pythagoras. Silk treats the "cosmological principle', the assumption that the Universe will look the same in all directions from all points within it, in considerable 
detail, its copernican foundations included. All of the modern stuff is there, among it a reasonably detailed description of why inflation is so compelling. Silk even has a chapter on 'alternative cosmologies', even though most of them are straw edifices which are easily pulled down. A reader of Silk's book will confront rather than avoid the peculiar properties of curved space which are characteristic of some cosmological models.

Nathan Cohen's Gravity's Lens falls short of the high standards of the others. His writing is too confusing, and he introduces too many terms which are underlined as though the reader has to remember them. You can understand how a particular type of star called a Cepheid variable can be used to measure the distance to galaxies without memorizing definitions of inverse square law, apparent magnitude, absolute magnitude and distance modulus - all of which are introduced in two pages! Too many factual slips mar the book (for example, quasars were identified as optical counterparts to radio sources and not, as Cohen says, as variable stars; it is the BL Lac objects which were first recognized as variables). On the good side, this book includes all the weird beasts that inhabit the astrophysical zoo and make the Universe such an interesting place. The treatment of gravitational lenses, which includes an account of the role of gadflies Jeno Barnothy and Fritz Zwicky in anticipating their discovery by decades, is decent enough.

Larry Marschall's supernova book is a superb piece of writing; it reads almost as easily as a good novel. Marschall's vivid descriptions bring astronomy to life. For instance, he reports that the radio telescope through which pulsars were discovered looked "like a giant colony of clothes lines waiting for wash day". He resists the temptation to pay undue attention to the recent supernova, and presents a balanced look at the whole field rather than a journalistic account of the life and times of supernova 1987A. He begins with a general treatment of astronomy, showing how supernovae fit into a bigger picture, and includes a very complete account of historical observations of these celestial explosions, in which stars suddenly blaze forth into the night-time, and even the day-time, sky, then fade away. The book is remarkably up to date - there is even a complete discussion of the mysterious spot and the light echoes which were discovered in mid-1988.

Chaisson's brief treatment of relativistic astrophysics is less successful, though one chapter, "Einstein, the Man", is pretty good. It is a slim collection of 30-second sound bites and pictures loosely organized about Einstein's theory of relativity and its astronomical consequences. Over-statements abound. For example, Chaisson writes that ". . . people from all segments of society seek to know whether the expansion of the Universe will continue for ever". He can't be serious. This question does not even count as a hot one for cosmologists in the $1980 \mathrm{~s}$, much less the average person; the inflationary model basically provides a postive answer. Indeed, the treatment of cosmology is rather dated, with little attention being paid to dark matter and the large-scale structure; it reads as though most of it were written ten years ago.

It seems to have become the fashion in books such as these to have few or no references either to other books or to the research literature. Although a popular

\section{Mathland on \$17.95}

\section{J. S. Clarke}

The Mathematical Tourist: Snapshots of Modern Mathematics. By Ivars Peterson. W. H. Freeman:1988. Pp.240. \$17.95, $£ 15.95$.

WE RESIDENTS have an ambivalent attitude to tourists: pleased that they are interested in the locality, irritated when they trample on the flowers. But Ivars Peterson behaves himself impeccably in his tour of the world of mathematics - one would almost think him a native - and his modestly subtitled collection of "snapshots" would serve as a good guidebook to the territory.

The emphasis is mostly on present-day culture rather than historical monuments, concentrating entirely on growth points in pure mathematics. Dramatic achievements of the past, even the recent past, such as the use of model theory in studying decidability in logic, are not explicitly included, nor are unsolved questions where there is little progress such as the Riemann hypothesis. The Poincaré conjecture, however, is given its proper place, along with the dramatic human interest of the failed Rêgo-Rourke attempt at proof.

The result is a very representative survey of those topics that have recently gained prominence. It should be accessible to quite a broad population, including the many readers who have been inoculated against all forms of mathematical reasoning by exposure to a de-natured form of it in their youth. Peterson succeeds in conveying a suprisingly good feel for the content of mathematics without, most of the time, actually doing any.

There is an inevitable bias towards the more spectacular and less abstract areas. Thus the account of the Mandelbrot set is one of the most successful parts of the book; but it is well placed in context, linked with the Julia sets and fully defined, and one forgives the few occasions when Peterson gets carried away by the graphics book does not demand the historian's detailed attention to footnotes and so on, some indications of further reading, or of where one can follow up a particular topic, are surely essential. Trefil includes a few passing references (but only to his own books), while Parker and Cohen have none at all. So here the plaudits go to Chaisson, Marschall and Silk, three authors who have taken the trouble to ensure that their books contain useful bibliographies.

Harry Shipman is Professor of Physics and Astronomy and Sharon Juska is an undergraduate in English at the University of Delaware, Newark, Delaware 19716, USA.

("iridescent dragons clawing their own tails ... and geometric jets spewing colored streams into still, black basins . . ."). At the other extreme of conceptual difficulty, he introduces the discovery of the non-standard differential structure on $\mathbb{R}^{+}$ by Donaldson. It is doubtful whether the concept of differential topology (as opposed to topology or geometry) will really get across to those not already familiar with it, but a valiant attempt is made.

A recurring theme is the use of computers. Indeed, if there is any 'message' in the book it is that computation is changing the nature of mathematics. Peterson shows computers as vital to the exploration of a variety of areas by providing visual representations of mathematical objects and so suggesting both new conjectures and ways of proving them; computers as themselves sources of mathematical problems, concerning the encryption of data, protocols for computer communication or the whole area of computational complexity; and computers as playing an actual part in the proof of theorems (though the four-colour theorem remains the only major instance of this).

There are frequent mentions of applications, exports from mathematics to other lands, but these tend to be unconvincing. the applications being of doubtful utility and seeming only palely to reflect the fervour of the research front. Many astronomers, for example, would question the assertion that "Astrophysicists have now confirmed that ... mass within the universe is distributed throughout space like a three-dimensional Cantor set", while the applications of knot theory to DNA structures involve little mathematics that was not available in the nineteenth century. The truth is that the real value of mathematics is not to be found in the latest Big Theorems, but in the whole integrated body of techniques and ideas that are needed to prove the theorems, and which they in turn give rise to.

C.J.S. Clarke is a Professor in the Departmen of Mathematics, University of Southampton. Southampton SO9 $5 \mathrm{NH}, U K$ K. 